\title{
SLC3A2 is upregulated in human osteosarcoma and promotes tumor growth through the PI3K/Akt signaling pathway
}

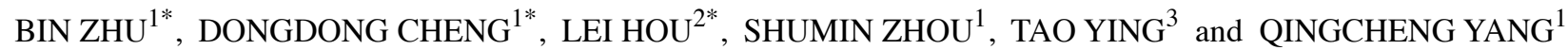 \\ ${ }^{1}$ Department of Orthopedics, Shanghai Jiao Tong University Affiliated Sixth People's Hospital, Shanghai 200233; \\ ${ }^{2}$ Department of Breast and Thyroid Surgery, The General Hospital of Jinan Military Command, Jinan, Shandong 116000; \\ ${ }^{3}$ Department of Ultrasound, Shanghai Jiao Tong University Affiliated Sixth People's Hospital, Shanghai 200233, P.R. China
}

Received September 25, 2016; Accepted November 10, 2016

DOI: 10.3892/or.2017.5530

\begin{abstract}
Growing evidence indicates that SLC3A2 (solute carrier family 3 member 2) is upregulated and correlates with tumor growth in multiple types of cancers, while the role of SLC3A2 in human osteosarcoma (OS) is rarely discussed. Thus, the aim of the present study was to demonstrate the expression of SLC3A2 in human osteosarcoma and reveal its biological function and the underlying mechanisms. RT-PCR, western blot analysis and immunohistochemistry (IHC) were used to assess the expression of SLC3A2 in OS samples and cell lines. Cell cycle, Cell Counting Kit-8 (CCK-8) and colony formation assays were used to test the cell survival capacity. To investigate the potential mechanism by which SLC3A2 regulates $\mathrm{OS}$ growth, we used a slide-based antibody array. We demonstrated that SLC3A2 was upregulated in OS cell lines as well as OS tissues. High expression of SLC3A2 was correlated with clinical stage and tumor size in OS. Reduced expression of SLC3A2 inhibited OS cell proliferation through G2/M phase arrest. Most importantly, we found that SLC3A2 may regulate OS growth through the PI3K/Akt signaling pathway. In conclusion, SLC3A2 is upregulated in OS and plays a crucial role in tumor growth. Targeting SLC3A2 may provide a new therapeutic strategy for OS.
\end{abstract}

Correspondence to: Dr Qingcheng Yang, Department of Orthopedics, Shanghai Jiao Tong University Affiliated Sixth People's Hospital, 600 Yishan Road, Shanghai 200233, P.R. China

E-mail: tjyqc@163.com

Dr Tao Ying, Department of Ultrasound, Shanghai Jiao Tong University Affiliated Sixth People's Hospital, 600 Yishan Road, Shanghai 200233, P.R. China

E-mail: yingtaomail@yeah.net

${ }^{*}$ Contributed equally

Key words: SLC3A2, CD98hc, osteosarcoma, proliferation

\section{Introduction}

Osteosarcoma (OS) is the most common primary bone malignancy in children, adolescents and young adults with a high tendency of local invasion and distant metastases $(1,2)$. The introduction of chemotherapy has led to a dramatic improvement in prognosis for patients with localized osteosarcoma; long-term survival rates of less than $20 \%$ improved to $65-70 \%$ after the advent of multi-agent chemotherapy regimens in the 1980s (3). However, in the last three decades, the efficacy of treatment has remained unchanged (4). Multiple factors are involved in the complex process of tumorigenesis (5), thus, uncovering the molecular mechanisms of OS may help to identify effective therapies for OS treatment.

Membrane transporters (MTs) are proteins that regulate the transport of endogenous molecules and xenobiotics across the cell membrane (6). Recently, MTs have attracted significant attention for their key roles in tumor growth, survival relapse and drug resistance $(7,8)$. MTs may be grouped into various classes, with the most important classes being ion channels (9), ABC transporters (10), water channels (11), pumps (such as sodium potassium pumps) (12) and the solute carriers (SLCs), which, by far, is the largest group of transporters (13).

SLC3A2 (solute carrier family 3 member 2), also known as CD98 heavy chain, is a type of SLC. Recent studies have reported that SLC3A2 is overexpressed and correlated with tumor progression in numerous human cancers, including head and neck squamous cell carcinoma (14), biliary tract cancer (15), non-small cell lung cancer (16), and triple-negative breast cancer (17). However, the role of SLC3A2 in OS progression is still unknown.

In this study, we report for the first time that SLC3A2 is upregulated in human OS. Additionally, we found that knockdown of SLC3A2 resulted in growth inhibition in OS cell lines and led to PI3K/Akt signaling pathway dysregulation, which indicates that SLC3A2 may play a crucial role in the growth of OS by influencing the PI3K/Akt signaling pathway.

\section{Materials and methods}

Cell lines and cell culture. The human osteosarcoma cell lines MNNG/HOS, MG63 and U2OS were used in this study. All cell lines were incubated at $37^{\circ} \mathrm{C}$ in a humidified 
atmosphere containing 5\% $\mathrm{CO}_{2}$. MNNG/HOS and MG63 cells were cultured in Dulbecco's modified Eagle's medium (DMEM) containing 10\% fetal bovine serum (FBS South America origin; Bio-West, Logan, UT, USA), $100 \mathrm{U} / \mathrm{ml}$ penicillin (Sigma-Aldrich, St. Louis, MO, USA) and $100 \mathrm{mg} / \mathrm{ml}$ streptomycin (Sigma-Aldrich), and U2OS cells were cultured in RPMI-1640 medium containing 10\% FBS, $100 \mathrm{U} / \mathrm{ml}$ penicillin and $100 \mathrm{mg} / \mathrm{ml}$ streptomycin.

Human osteosarcoma samples. In total, 50 human osteosarcoma samples and the adjacent non-cancerous tissues were obtained from patients who underwent treatment at the Department of Orthopedics, Shanghai Jiao Tong University Affiliated Sixth People's Hospital (Shanghai, China) between 2013 and 2014. To avoid deviation from the heterogeneity of the tumor body, we obtained tumor tissue samples from several sections of the tumor. More than $80 \%$ of cancerous section were obtained. The samples were frozen and stored in liquid nitrogen after resection. All the procedures were approved by the Ethics Committee of the Shanghai Jiao Tong University Affiliated Sixth People's Hospital and under consensus agreement.

RNA isolation and qRT-PCR assays. The total RNA of cells and human tissue samples was extracted using TRIzol reagent (Invitrogen, Carlsbad, CA, USA) and quantified by Nanodrop 2000 (Thermo Fisher Scientific, Waltham, MA, USA). The first-stand cDNA was synthesized using the PrimeScript RT reagent kit (Takara, Shiga, Japan) and was used as a template for the PCR detection. RT-PCR was performed with SYBR Green Premix Ex Taq (Takara) according to the manufacturer's protocol. All reactions were performed three times in a $10-\mu 1$ reaction volume. The expression level of genes was measured using the comparative $\mathrm{Ct}$ method. The information of the primer sequences used was described as follows: SLC3A2 forward, ACCCCTGTTTTCAGCTACGG and reverse, GGTC TTCACTCTGGCCCTTC; $\beta$-actin forward, TTGTTACA GGAAGTCCCTTGCC and reverse, ATGCTATCACCTCC CCTGTGTG.

Cell transfection. The siRNA targeting SLC3A2 as well as a negative control were synthesized by RiboBio Co., Ltd. (Guangzhou, China). The siRNA sequences targeting SLC3A2 were: forward primer, 5'-AGAUGAAGAUAGUCAAGAA-3', and reverse primer, 5'-UUCUUGACUAUCUUCAUCU-3'. RNAi-Max (Invitrogen) was used in the transfection. All the steps were applied in accordance with the manual.

Cell proliferation and cell cycle assays. For cell proliferation assay, 3000 cells transfected with targeted siRNA or si-NC were seeded in each well of a 96-well microplate 2 days after the transfection. To measure the cell viability, we added $10 \mu \mathrm{l}$ Cell Counting Kit-8 (CCK-8, Dojindo Molecular Technologies, Inc., Kumamoto, Japan) and $100 \mu 1$ DMEM (for MNNG/HOS) or RPMI-1640 (for U2OS) to each well and incubated for $2 \mathrm{~h}$. The absorbance at a wavelength of $450 \mathrm{~nm}$ was measured by a microplate reader (Model 680, Bio-Rad Laboratories, Hercules, CA, USA). For cell cycle assay, cells were fixed in $70 \%$ ethanol for $24 \mathrm{~h}$ at $-20^{\circ} \mathrm{C}$ two days after the transfection. Then, staining solution containing $50 \mu \mathrm{g} / \mathrm{ml}$ RNase A (BD
LSRII, San Jose, CA, USA) and $50 \mu \mathrm{g} / \mathrm{ml}$ propidium iodide (PI) (Biolegend, San Diego, CA, USA) was used to treat the cells. All the experiments were performed three times.

Colony formation assay. OS cells transfected with targeted siRNA or si-NC were collected 2 days after the transfection and were planted in 6 -well plates at a density of 1000 cell/well. The cells were cultured for 2 weeks at a constant temperature of $37^{\circ} \mathrm{C}$ in a humidified atmosphere containing $5 \% \mathrm{CO}_{2}$ to form colonies. Colonies were fixed in $95 \%$ methanol and stained with $0.1 \%$ crystal violet for $20 \mathrm{~min}$, respectively. The colonies were then counted and photographed. The assay was repeated three times.

Western blot analysis and PathScan intracellular signaling array kit. A mixture of PhosSTOP (Roche, Basel, Switzerland), T-PER protein extraction reagent (Thermo Fisher Scientific) and Complete Mini (Roche) was used to extract the cell lysates. Equal amounts of protein $(20 \mu \mathrm{g})$ were loaded and separated using $8 \%$ sodium dodecyl sulfate polyacrylamide gel electrophoresis (SDS-PAGE) and then transferred to nitrocellulose membranes (Millipore). After blocking in 5\% milk with phosphate-buffered saline for $1 \mathrm{~h}$, the membranes were incubated with primary antibodies listed below at $4^{\circ} \mathrm{C}$ overnight: SLC3A2 (Proteintech, 1:500), PI3-kinase (Phospho-Tyr607) (Signalway Antibody, 1:500), Akt (Phospho-Ser473, 1:500) or $\beta$-actin (Sigma-Aldrich, 1:20000). The secondary antibody was anti-rabbit IgG (Sigma-Aldrich, 1:5000). Next, the detection of the blots was performed using SuperSignal West Femto Maximum Sensitivity Substrate (Thermo Fisher Scientific). To analyze the signaling pathways influenced by SLC3A2 expression, we used PathScan Intracellular Signaling Array kit (Cell Signaling Technology). This system is a slide-based antibody array, based upon the sandwich immunoassay principle and allowing for the simultaneous detection of 18 important well-characterized signaling molecules when phosphorylated or cleaved. The experiment was performed according to the manufacturer's instructions, and the fluorescent images of the slide were then captured with Image Studio Software (Licensor Inc.). Images were analyzed with ImageJ software. Normalization was performed by subtracting the intensity of the negative control dot from each value.

Immunohistochemistry (IHC). As previously described (18), immunohistochemistry was performed with the following steps: After being warmed in a $60^{\circ} \mathrm{C}$ oven, de-waxed in three changes of xylene, and passaged through graded ethanol (100, 95, and 70\%), formalin-fixed and paraffin-embedded sections came to a final wash in distilled water. Hydrogen peroxide (3\%) was used to quench the endogenous peroxidase acivity and BSA was used to block for $30 \mathrm{~min}$. The sections were incubated with an antibody against SLC3A2 at a 1:50 dilution overnight in $4^{\circ} \mathrm{C}$. The slides were mounted for microscopic evaluation and photographed after being counterstained with Gill's hematoxylin for $1 \mathrm{~min}$ and dehydrated. The staining results for SLC3A2 protein were scored according to an established 0-3 scale as listed below: 0, no staining; 1 , staining in $<1 \%$ cells; 2 , staining in $1-10 \%$ cells; 3 , staining in $>10 \%$ of cells. We considered the samples graded 0 and 1 as negative, and those graded as 2 and 3 as positive. In total, 10 optical 


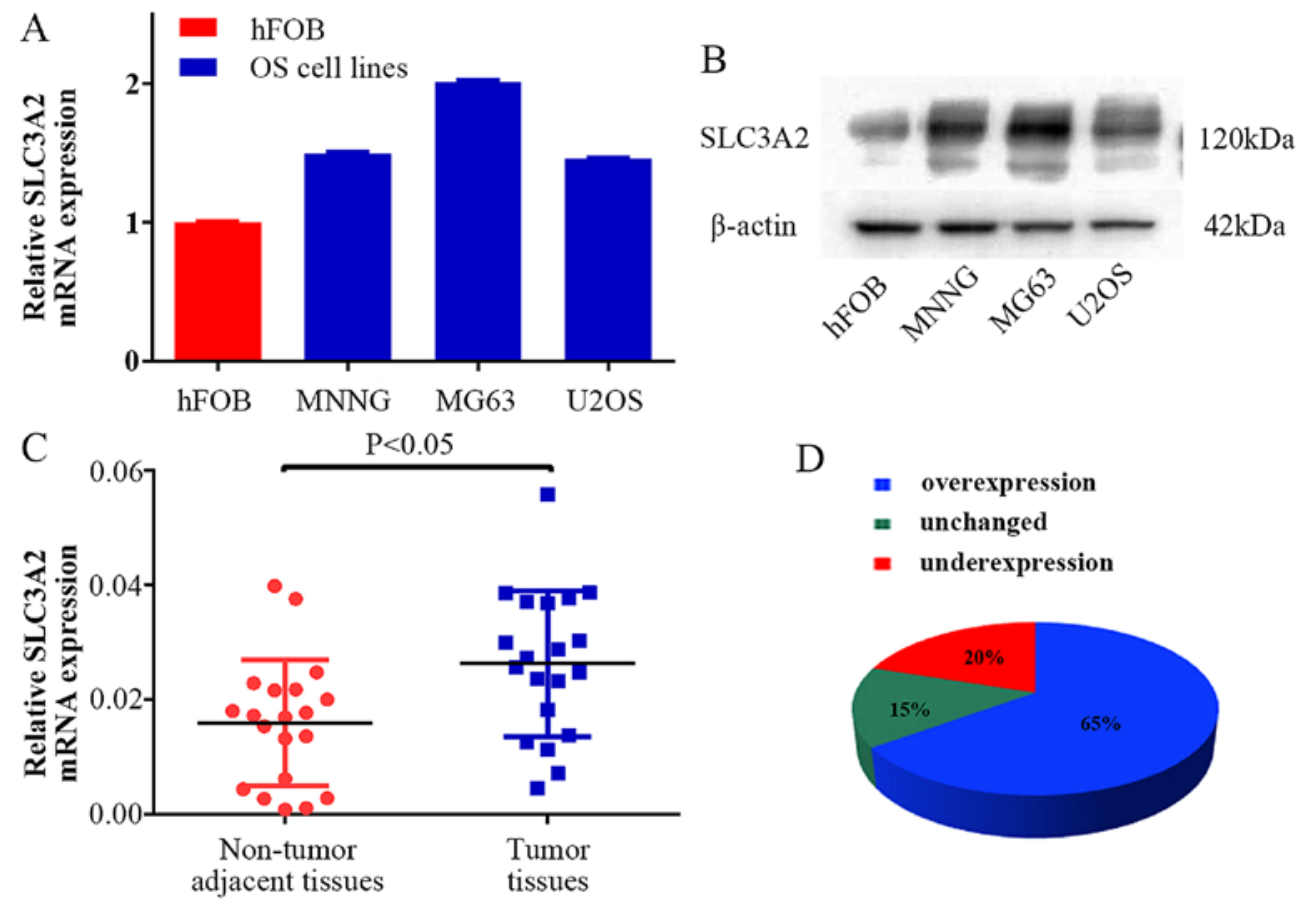

Figure 1. SLC3A2 expression is upregulated in OS clinical samples and cell lines. (A and B) The mRNA and protein expression levels of SLC3A2 were measured by TaqMan real-time PCR and western blot assays in OS cell lines (including MNNG/HOS, MG63 and U2OS) and a human osteoblastic cell line ( $\mathrm{hFOB}$ ). (C and D) Relative expression of SLC3A2 was detected using qRT-PCR in 20 pairs of OS samples and their corresponding non-cancerous samples. Statistical analysis was performed using paired t-test in C.

fields from three different sections were used for each evaluation by two experienced colleagues, respectively.

Statistical analysis. Data were analyzed with SPSS software (version 20.0) (IBM Corp., Armonk, NY, USA). Student's t-test was used to compare the differences between groups. Pearson's Chi-square or Continuity Correction test was used to calculate the correlations between parameters. All the data were graphically imaged by GraphPad Prism 5 software (Graphpad Software, Inc., La Jolla, CA, USA).

\section{Results}

Expression of SLC $3 A 2$ is upregulated in OS clinical samples and cell lines. To clarify the expression level of SLC3A2 in OS, we examined SLC3A2 expression at the mRNA and protein levels, respectively. As a result, higher expression of SLC3A2 was observed in the OS cell lines (MNNG/HOS, MG63, U2OS) compared with that noted in the human osteoblast cell line (hFOB) (Fig. 1A and B). Moreover, the expression of SLC3A2 in 20 pairs of human OS tissue samples and their corresponding non-cancerous tissue controls were also analyzed using quantitative RT-PCR (qRT-PCR). As shown in Fig. 1C and D, SLC3A2 expression was significantly upregulated in tumor tissue samples compared to that noted in the corresponding controls $(65 \%$, $13 / 20)$.

Knockdown of SLC $3 A 2$ leads to reduced proliferation and cell cycle arrest in the $G 2 / M$ phase. To ascertain the underlying role of SLC3A2 in tumorigenesis, we first evaluated the effect of SLC $3 \mathrm{~A} 2$ on the growth of OS cells in vitro. After knockdown of SLC3A2 by specifically targeted siRNA (Fig. 2A and B), the viability of the MNNG/HOS and U2OS cells was clearly inhibited, as determined by CCK-8 assay (Fig. 2C and D). Cell cycle distribution was analyzed by flow cytometry after transfection with si-NC or si-SLC3A2. The results of the cell cycle analysis revealed that knockdown of SLC3A2 expression resulted in G2/M phase arrest in both the MNNG/HOS and U2OS cell lines (Fig. 2E-H). Thus, these results indicate that SLC3A2 is essential for the proliferation of OS cells.

Silencing of SLC3A2 attenuates colony formation capacity of OS cells. To further elucidate the potential role of SLC3A2 in tumorigenesis, we determined whether the knockdown of SLC3A2 expression affects the colony formation ability of OS cells by colony formation assay. As a result, both the size and the number of colonies were markedly reduced in the SLC3A2-knockdown group compared with these parameters in the control group (Fig. 3A and C). The number of colonies was significantly reduced by 68.5 and $69.3 \%$ in the MNNG/ HOS and U2OS cells, respectively (Fig. 3B and D). Taken together, these results demonstrated that SLC3A2 is important for OS cell growth.

SLC3A2 regulates the PI3K/AKT signaling pathway in OS cells. To further seek the potential molecular mechanism through which knockdown of SLC3A2 expression suppresses OS cell proliferation, we performed a signaling antibody array assay. As shown in Fig. 4A-D, knockdown of SLC3A2 expression led to reduced expression of $\mathrm{p}-\mathrm{AKT}$ in the $\mathrm{MNNG} / \mathrm{HOS}$ and U2OS cell lines, suggesting that SLC3A2 may promote OS cell proliferation through the PI3K/AKT signaling pathway. These results were further supported by western blot analysis 
A MNNG/HOS

B $\mathrm{U} 2 \mathrm{OS}$
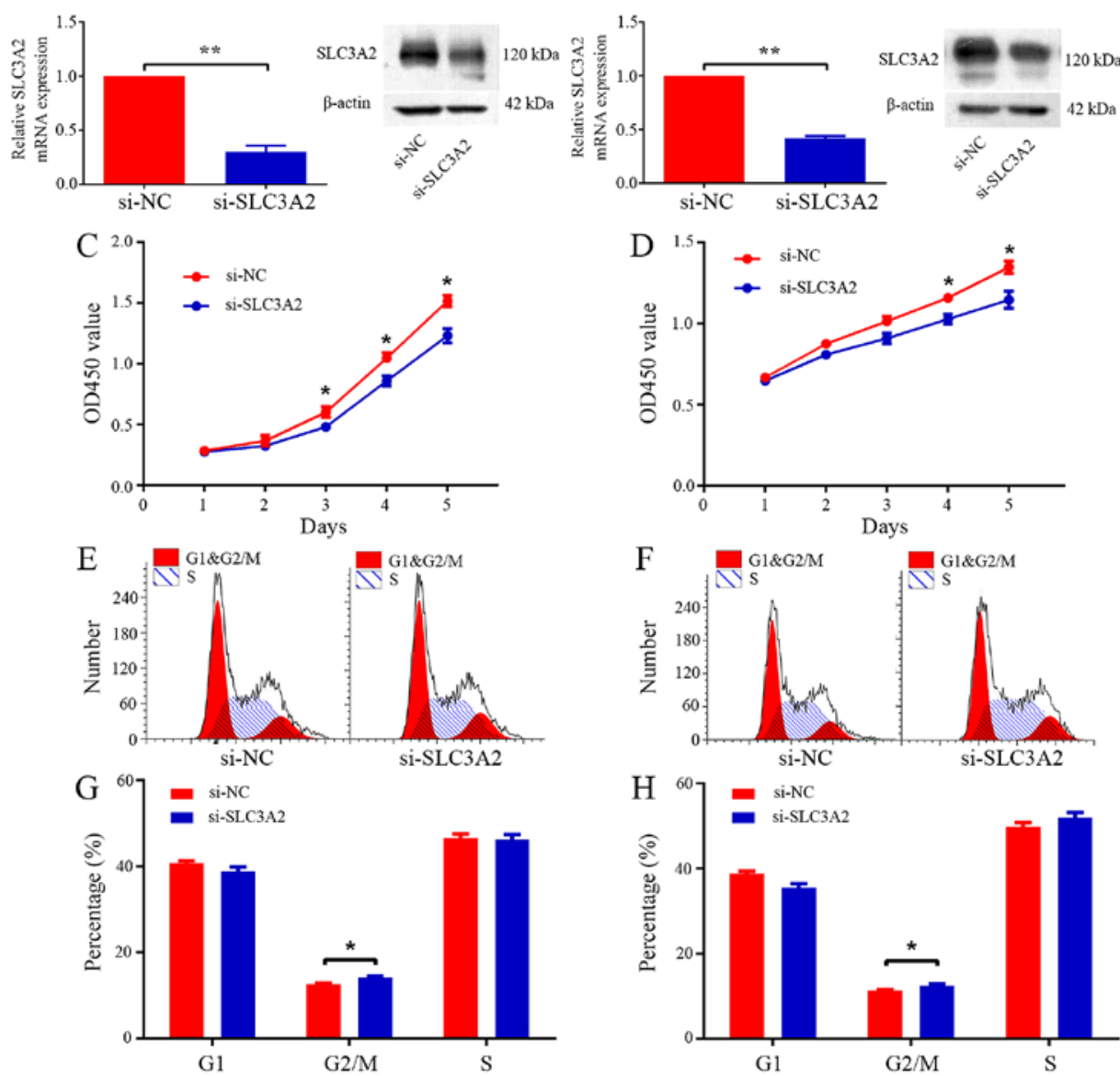

Figure 2. Knockdown of SLC3A2 inhibits cell proliferation through G2/M phase arrest. (A and B) The expression of SLC3A2 was downregulated by a targeted siRNA. (C and D) Diagrams showing results of the CCK-8 assay. MNNG/HOS and U2OS cell proliferation was inhibited by downregulation of SLC3A2 expression. Cell cycle profiles determined by propidium iodide (PI) staining and flow cytometry assays of (E and G) MNNG/HOS and (F and H) U2OS cells transfected with si-SLC3A2 or si-NC. Data are representative of three independent experiments. Error bars represent SD. " $\mathrm{P}<0.05,{ }^{* *} \mathrm{P}<0.01$ by Student's t-test in A-D and Wilcoxon signed rank sum test in $\mathrm{G}$ and $\mathrm{H}$.

A

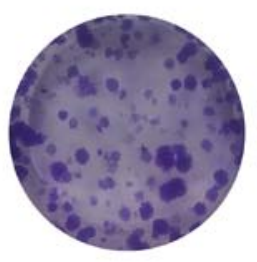

si-NC

C

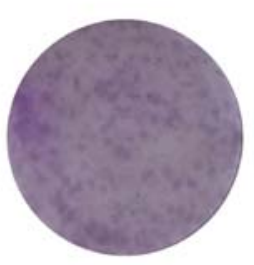

si-NC
MNNG/HOS

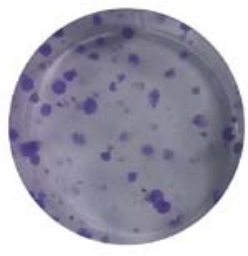

si-SLC3A2

$20 \mathrm{~S}$

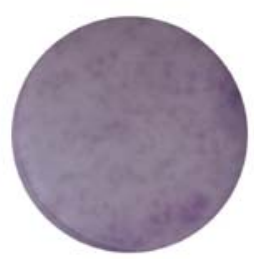

si-SLC3A2
B

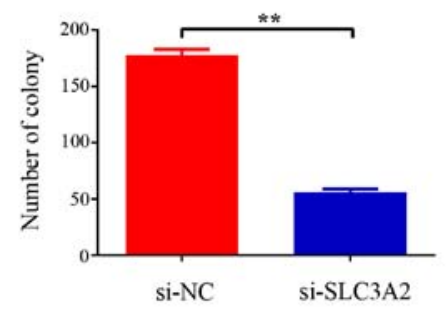

$\mathrm{D}$

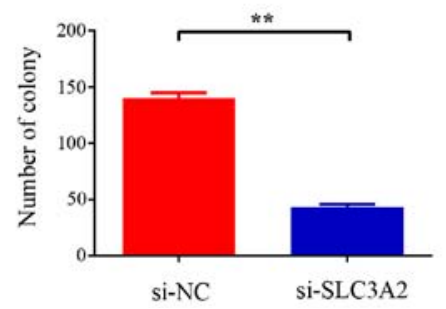

Figure 3. Decrease in SLC3A2 impairs OS cell colony formation capacity. (A and C) Representative photomicrographs of (A) MNNG/HOS and (C) U2OS cell colonies in culture plates. (B and D) Significant reduction in the colony formation efficacy in (B) MNNG/HOS and (D) U2OS cells following SLC3A2 knockdown. Data are expressed as the mean \pm SD of three independent experiments. ${ }^{* *} \mathrm{P}<0.01$, by student's t-test. 
A

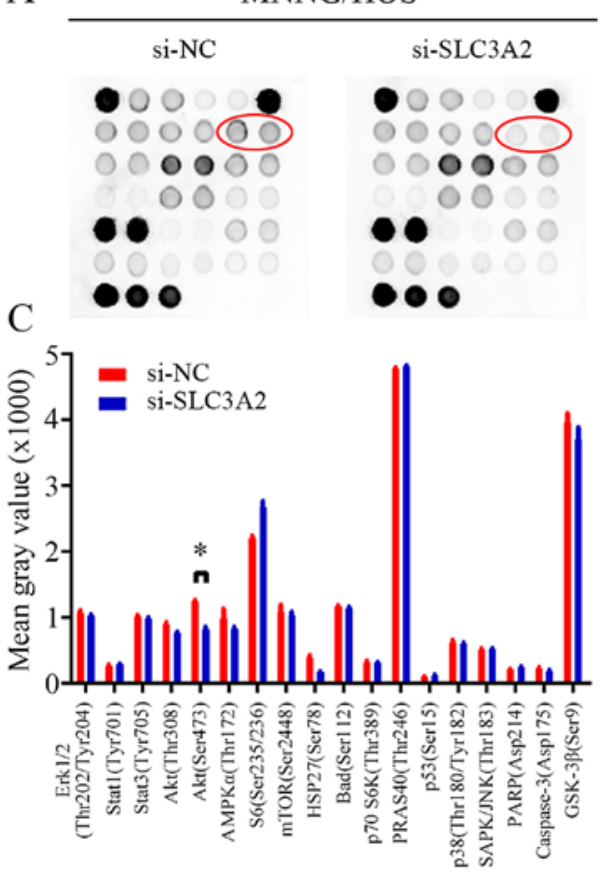

E
B

D

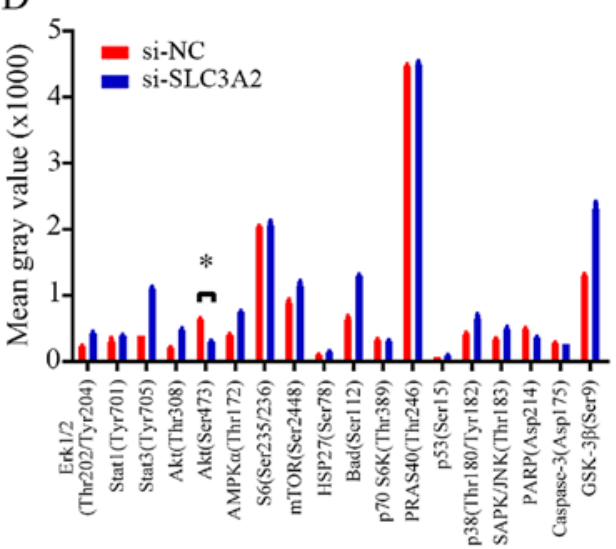

U2OS

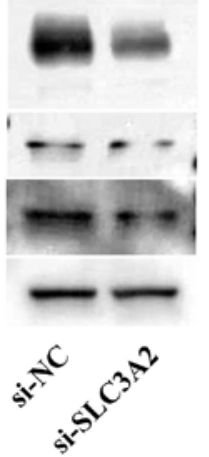

$120 \mathrm{kDa}$

80 kDa

$60 \mathrm{kDa}$

$42 \mathrm{kDa}$

Figure 4. SLC3A2 regulates the PI3K/AKT signaling pathway. (A and B) PathScan Intracellular Signaling array kit was used to analyze the signaling pathways influenced by knockdown of SLC3A2 expression in the MNNG/HOS and U2OS cells. (C and D) Diagrams showing the mean gray value of 18 key signaling molecules revealed that the p-Akt (Ser473) is downregulated by knockdown of SLC3A2 expression in both the MNNG/HOS and U2OS cell lines. (E) Western blot analysis of p-PI3K (Tyr607) and p-Akt (Ser473) in MNNG/HOS and U2OS cells transfected with si-NC and si-SLC3A2. Data are representative of three independent experiments. ${ }^{*} \mathrm{P}<0.05$, by Student's t-test.

as shown in Fig. 4E, where the expression of PI3K and AKT were both downregulated by inhibition of SLC3A2.

High expression of SLC3A2 correlates with OS clinical stage and tumor size. We performed immunohistochemical staining on 50 human osteosarcoma tissues and their corresponding non-cancerous tissue controls. Representative examples of IHC for SLC3A2 in the OS and non-cancerous tissues are shown in Fig. 5. The positive rate of IHC among OS and non-cancerous tissues was 58\% (29/50) and 14\% (7/50), respectively. In addition, $62 \%$ (31/50) of the matched cases showed high SLC3A2 expression in the OS tissues compared to that observed in the non-cancerous tissues. We then conducted stratified analyses to assess SLC3A2 expression in the OS patients with specific clinical characteristics as shown in Table I. While SLC3A2 expression was not correlated with age, gender, anatomic location, degree of malignancy or tumor necrosis rate, there were significant correlations with clinical stage $(\mathrm{P}<0.05)$ and tumor size $(\mathrm{P}<0.05)$ which achieved statistical significance. Taken together, these results indicate that SLC3A2 is upregulated and potentially plays a pivotal role in the growth and survival of OS.

\section{Discussion}

According to current knowledge, SLC proteins can translocate diverse endogenous substrates, drugs and environmental toxicants. As a result, they play crucial roles in the development of tumor progression and therapy (19). Multiple SLC transporters have been investigated in recent studies. A study by Babu et al demonstrated that SLC6A14 is crucial for the maintenance of amino acid nutrition and optimal mammalian target of rapamycin (mTOR) signaling in $\mathrm{ER}^{+}$breast cancer (20). Chen et al reported that genomic polymorphisms of SLC29A3 are associated with overall survival in advanced non-small cell lung cancer treated with gemcitabine (21). Salaün et al revealed that SLC20A1 has a direct biological role in the downstream apoptotic signaling pathway induced by tumor necrosis factor (TNF) (22). 


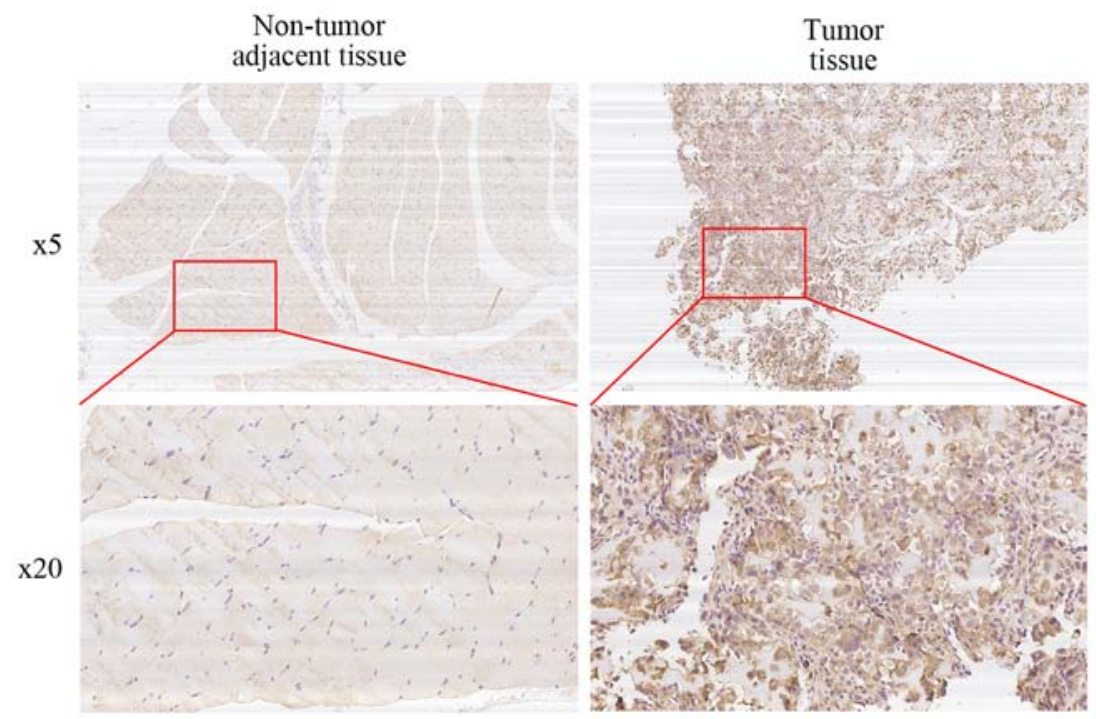

Figure 5. High expression of SLC3A2 is correlated with OS clinical stage and tumor size. Representative micrographs are shown. SLC3A2 is overexpressed in OS tissues compared with that observed in corresponding non-cancerous tissues.

Table I. Correlation of the immunohistochemical staining (IHC) for SLC3A2 and clinicopathologic parameters in 50 osteosarcoma patients.

\begin{tabular}{|c|c|c|c|c|c|c|c|}
\hline \multirow{2}{*}{$\begin{array}{l}\text { Clinicopathologic } \\
\text { parameters }\end{array}$} & \multirow{2}{*}{$\begin{array}{l}\text { Number } \\
\text { of cases }\end{array}$} & \multicolumn{2}{|c|}{ IHC of SLC3A2 } & \multirow[b]{2}{*}{$\chi^{2}$} & \multirow[b]{2}{*}{ P-value } & \multirow[b]{2}{*}{ Odds ratio } & \multirow[b]{2}{*}{$95 \% \mathrm{CI}$} \\
\hline & & Positive & Negative & & & & \\
\hline \multicolumn{8}{|l|}{ Age (years) } \\
\hline$<20$ & 31 & 19 & 12 & 0.363 & 0.547 & - & - \\
\hline$\geq 20$ & 19 & 10 & 9 & & & & \\
\hline \multicolumn{8}{|l|}{ Gender } \\
\hline Male & 27 & 13 & 14 & 2.339 & 0.126 & - & - \\
\hline Female & 23 & 16 & 7 & & & & \\
\hline \multicolumn{8}{|l|}{ Anatomic location } \\
\hline Tibia/ Femur & 33 & 21 & 12 & 1.266 & 0.261 & - & - \\
\hline Elsewhere & 17 & 8 & 9 & & & & \\
\hline \multicolumn{8}{|l|}{ Enneking stage } \\
\hline $\mathrm{I} / \mathrm{II}$ & 35 & 16 & 19 & 5.645 & $0.018^{\mathrm{a}}$ & 7.719 & $1.512-39.42$ \\
\hline III & 15 & 13 & 2 & & & & \\
\hline \multicolumn{8}{|l|}{ Tumor size $\left(\mathrm{cm}^{3}\right)$} \\
\hline$<50$ & 21 & 8 & 13 & 5.889 & $0.015^{\mathrm{a}}$ & 4.266 & $1.285-14.16$ \\
\hline$\geq 50$ & 29 & 21 & 8 & & & & \\
\hline \multicolumn{8}{|c|}{ Degree of malignancy } \\
\hline Low & 23 & 10 & 13 & 3.687 & 0.055 & - & - \\
\hline High & 27 & 19 & 8 & & & & \\
\hline \multicolumn{8}{|c|}{ Tumor necrosis rate (\%) } \\
\hline$<90$ & 27 & 17 & 10 & 0.593 & 0.441 & - & - \\
\hline$\geq 90$ & 23 & 12 & 11 & & & & \\
\hline
\end{tabular}

P-value represents the probability from Pearson's Chi-square or Continuity Correction test for IHC of SLC3A2 expression between variable subgroups. ${ }^{a} \mathrm{P}<0.05$, a significant difference. $\mathrm{CI}$, confidence interval.

In the present study, we demonstrated that SLC3A2, one of the SLC transporters, was significantly upregulated in OS clinical samples compared with that noted in non-cancerous tissues. Moreover, we found that the expression of SLC3A2 was correlated with OS Enneking stage and tumor size according to IHC staining. To date, upregulation of SLC 3 A2 has been 
found in several different tumor types. Garber et al examined the global gene expression profiles of 67 human lung tumors and found that SLC3A2 was overexpressed in adenocarcinomas of the lung (23). Prager et al reported increased SLC3A2 expression in different types of malignant renal cell cancers (RCCs), lack of expression in benign renal oncocytoma and the extent of expression were correlated directly with the grade of malignancy (24). Yang et al confirmed the upregulation of SLC3A2 in gastric cancer cells by immunoblotting of a panel of 13 gastric cancer cell lines and immunohistochemistry of tissue microarrays comprising 85 matched pairs of normal and tumor tissues (25). However, the association of SLC3A2 expression with OS has not yet been reported.

To investigate the function of SLC3A2 in OS cells, we suppressed its expression using a targeted siRNA. Our experimental data revealed that knockdown of SLC3A2 inhibited cell proliferation through $\mathrm{G} 2 / \mathrm{M}$ phase arrest, and impaired OS cell colony formation capacity. Taken together, these results indicate that SLC3A2 is essential for OS cell survival and acts as an oncogene in OS. Consistently, SLC3A2 has been reported to enhance proliferation in other types of cells. $\mathrm{Wu}$ et al recently indicated that SLC3A2 was highly expressed and co-localized with basigin on the human hepatocellular carcinoma (HCC) cell membrane, and plays a critical role in promoting cell spreading and the progression of hepatocellular carcinoma (26). SLC3A2 is also required for proliferation of B and T lymphocytes $(27,28)$, vascular smooth muscle cells (29) and intestinal epithelial cells (30).

It was previously reported that SLC3A2 is an integrinassociated protein and contributes to integrin-dependent cell spreading, cell migration, and protection from apoptosis through activation of Akt and Rac GTPase, major contributors to integrin-dependent signals involved in cell survival and cell migration (31). Bulus et al demonstrated that overexpression of SLC3A2 increased renal tubular epithelial cell proliferation through Erk and p38 MAPK signaling pathway (32). To further seek the molecular mechanism by which SLC3A2 mediates OS cell proliferation, we used a slide-based antibody array, which allows for the simultaneous detection of 18 important and well-characterized signaling molecules when phosphorylated or cleaved $(33,34)$. Reduced expression of Akt (Ser473) was found after knockdown of SLC3A2 in both MNNG/ HOS and U2OS cell lines. We then considered the PI3K/ Akt signaling pathway, which is crucial for cell growth and survival (35). Western blotting further affirmed our hypothesis that SLC3A2 influences OS progression by regulating the PI3K/Akt signaling pathway.

In conclusion, we demonstrated in the present study for the first time that SLC3A2 was upregulated in OS cell lines and OS tissues, and that SLC3A2 expression was correlated with tumor size and clinical stage of human OS. Knockdown of the expression of SLC3A2 inhibited OS cell proliferation through the PI3K/Akt signaling pathway. Importantly, these findings provide insight into a novel therapeutic target for OS.

\section{References}

1. Moore DD and Luu HH: Osteosarcoma. Cancer Treat Res 162 : 65-92, 2014.
2. He JP, Hao Y, Wang XL, Yang XJ, Shao JF, Guo FJ and Feng JX: Review of the molecular pathogenesis of osteosarcoma. Asian Pac J Cancer Prev 15: 5967-5976, 2014.

3. Isakoff MS, Bielack SS, Meltzer P and Gorlick R: Osteosarcoma: Current treatment and a collaborative pathway to success. J Clin Oncol 33: 3029-3035, 2015.

4. Allison DC, Carney SC, Ahlmann ER, Hendifar A, Chawla S, Fedenko A, Angeles C and Menendez LR: A meta-analysis of osteosarcoma outcomes in the modern medical era. Sarcoma 2012: 704872, 2012.

5. Baumhoer D: Molecular characterization of osteosarcomas. Pathologe 34 (Suppl 2): 260-263, 2013 (In German).

6. Ho RH and Kim RB: Transporters and drug therapy: Implications for drug disposition and disease. Clin Pharmacol Ther 78: 260-277, 2005.

7. Abdulhussein AA and Wallace HM: Polyamines and membrane transporters. Amino Acids 46: 655-660, 2014.

8. Zinzi L, Contino M, Cantore M, Capparelli E, Leopoldo M and Colabufo NA: ABC transporters in CSC membranes as a novel target for treating tumor relapse. Front Pharmacol 5: 163, 2014.

9. Yu FH and Catterall WA: The VGL-chanome: A protein superfamily specialized for electrical signaling and ionic homeostasis. Sci STKE 2004: re15, 2004.

10. Montanari F and Ecker GF: Prediction of drug-ABC-transporter interaction - Recent advances and future challenges. Adv Drug Deliv Rev 86: 17-26, 2015.

11. Wang F, Feng XC, Li YM, Yang H and Ma TH: Aquaporins as potential drug targets. Acta Pharmacol Sin 27: 395-401, 2006.

12. Dunbar LA and Caplan MJ: Ion pumps in polarized cells: Sorting and regulation of the $\mathrm{Na}^{+}, \mathrm{K}^{+}$- and $\mathrm{H}^{+}, \mathrm{K}^{+}$-ATPases. $\mathrm{J}$ Biol Chem 276: 29617-29620, 2001.

13. Fredriksson R, Nordström KJ, Stephansson O, Hägglund MG and Schiöth HB: The solute carrier (SLC) complement of the human genome: Phylogenetic classification reveals four major families. FEBS Lett 582: 3811-3816, 2008.

14. Martens-de Kemp SR, Brink A, Stigter-van Walsum M, Damen JM, Rustenburg F, Wu T, van Wieringen WN, Schuurhuis GJ, Braakhuis BJ, Slijper M, et al: CD98 marks a subpopulation of head and neck squamous cell carcinoma cells with stem cell properties. Stem Cell Res (Amst) 10: 477-488, 2013.

15. Kaira K, Sunose Y, Oriuchi N, Kanai Y and Takeyoshi I: CD98 is a promising prognostic biomarker in biliary tract cancer. Hepatobiliary Pancreat Dis Int 13: 654-657, 2014.

16. Fei F, Li X, Xu L, Li D, Zhang Z, Guo X, Yang H, Chen Z and Xing J: CD147-CD98hc complex contributes to poor prognosis of non-small cell lung cancer patients through promoting cell proliferation via the PI3K/Akt signaling pathway. Ann Surg Oncol 21: 4359-4368, 2014.

17. Furuya M, Horiguchi J, Nakajima H, Kanai Y and Oyama T: Correlation of L-type amino acid transporter 1 and CD98 expression with triple negative breast cancer prognosis. Cancer Sci 103: 382-389, 2012.

18. Lin S, Guo Q, Wen J, Li C, Lin J, Cui X, Sang N and Pan J: Survival analyses correlate stanniocalcin 2 overexpression to poor prognosis of nasopharyngeal carcinomas. J Exp Clin Cancer Res 33: 26, 2014.

19. He L, Vasiliou K and Nebert DW: Analysis and update of the human solute carrier (SLC) gene superfamily. Hum Genomics 3: 195-206, 2009.

20. Babu E, Bhutia YD, Ramachandran S, Gnanaprakasam JP, Prasad PD, Thangaraju M and Ganapathy V: Deletion of the amino acid transporter Slc6a14 suppresses tumour growth in spontaneous mouse models of breast cancer. Biochem J 469: $17-23,2015$.

21. Chen X, Zhang L, Ren S, Li X, Zhou F, Li W, Gao G, He Y and Zhou C: Genomic polymorphisms of SLC29A3 associated with overall survival in advanced non-small-cell lung cancer treated with gemcitabine. Med Oncol 31: 865, 2014.

22. Salaün C, Leroy C, Rousseau A, Boitez V, Beck L and Friedlander G: Identification of a novel transport-independent function of PiT1/SLC20A1 in the regulation of TNF-induced apoptosis. J Biol Chem 285: 34408-34418, 2010.

23. Garber ME, Troyanskaya OG, Schluens K, Petersen S, Thaesler Z, Pacyna-Gengelbach M, van de Rijn M, Rosen GD, Perou CM, Whyte RI, et al: Diversity of gene expression in adenocarcinoma of the lung. Proc Natl Acad Sci USA 98: 13784-13789, 2001.

24. Prager GW, Poettler M, Schmidinger M, Mazal PR, Susani M, Zielinski CC and Haitel A: CD98hc (SLC3A2), a novel marker in renal cell cancer. Eur J Clin Invest 39: 304-310, 2009. 
25. Yang Y, Toy W, Choong LY, Hou P, Ashktorab H, Smoot DT, Yeoh KG and Lim YP: Discovery of SLC3A2 cell membrane protein as a potential gastric cancer biomarker: Implications in molecular imaging. J Proteome Res 11: 5736-5747, 2012.

26. Wu B, Wang Y, Yang XM, Xu BQ, Feng F, Wang B, Liang Q, Li Y, Zhou Y, Jiang JL, et al: Basigin-mediated redistribution of CD98 promotes cell spreading and tumorigenicity in hepatocellular carcinoma. J Exp Clin Cancer Res 34: 110, 2015.

27. Cantor J, Browne CD, Ruppert R, Féral CC, Fässler R, Rickert RC and Ginsberg MH: CD98hc facilitates B cell proliferation and adaptive humoral immunity. Nat Immunol 10: 412-419, 2009.

28. Cantor J, Slepak M, Ege N, Chang JT and Ginsberg MH: Loss of $\mathrm{T}$ cell CD98 $\mathrm{H}$ chain specifically ablates $\mathrm{T}$ cell clonal expansion and protects from autoimmunity. J Immunol 187: 851-860, 2011.

29. Fogelstrand P, Féral CC, Zargham R and Ginsberg MH: Dependence of proliferative vascular smooth muscle cells on CD98hc (4F2hc, SLC3A2). J Exp Med 206: 2397-2406, 2009.

30. Nguyen HT, Dalmasso G, Torkvist L, Halfvarson J, Yan Y, Laroui H, Shmerling D, Tallone T, D'Amato M, Sitaraman SV, et al: CD98 expression modulates intestinal homeostasis, inflammation, and colitis-associated cancer in mice. J Clin Invest 121 1733-1747, 2011
31. Feral CC, Nishiya N, Fenczik CA, Stuhlmann H, Slepak M and Ginsberg MH: CD98hc (SLC3A2) mediates integrin signaling. Proc Natl Acad Sci USA 102: 355-360, 2005.

32. Bulus N, Feral C, Pozzi A and Zent R: CD98 increases renal epithelial cell proliferation by activating MAPKs. PLoS One 7: e40026, 2012.

33. Montero JC, Esparís-Ogando A, Re-Louhau MF, Seoane S, Abad M, Calero R, Ocaña A and Pandiella A: Active kinase profiling, genetic and pharmacological data define mTOR as an important common target in triple-negative breast cancer. Oncogene 33: 148-156, 2014.

34. Yao W, Guan M, Jia J, Dai W, Lay YA, Amugongo S, Liu R, Olivos D, Saunders M, Lam KS, et al: Reversing bone loss by directing mesenchymal stem cells to bone. Stem Cells 31: 2003-2014, 2013.

35. Porta C, Paglino C and Mosca A: Targeting PI3K/Akt/mTOR Signaling in Cancer. Front Oncol 4: 64, 2014. 